\title{
Lean and green? Evidências empíricas do setor automotivo brasileiro
}

\author{
Lean and green? Empirical evidence from the brazilian \\ automotive industry
}

\author{
Ana Beatriz Lopes de Sousa Jabbour ${ }^{1}$ \\ Charbel José Chiappetta Jabbour ${ }^{1}$ \\ Wesley Ricardo de Souza Freitas ${ }^{2}$ \\ Adriano Alves Teixeira ${ }^{3}$
}

\begin{abstract}
Resumo: O objetivo deste artigo é verificar se as práticas de manufatura enxuta estão se relacionando positivamente com a adoção de práticas de gestão ambiental em empresas brasileiras do setor automotivo, com a finalidade de testar uma hipótese largamente argumentada na literatura de que ao ser "lean", uma empresa tende também a ser "green". Para tanto, foi sugerido um framework relacionando esses conceitos, com a proposição de uma hipótese de pesquisa. Os dados, coletados em 75 empresas, foram analisados por meio de Modelagem de Equações Estruturais. Os principais resultados indicam que a manufatura enxuta (LM) se relaciona positivamente à gestão ambiental (EM), mas o poder de determinação de LM em EM tende a ser considerado entre fraco e moderado. De fato, ser lean é ser também green, mas outras variáveis organizacionais não consideradas neste estudo podem estar influenciando o greening das organizações.
\end{abstract}

Palavras-chave: Gestão ambiental. Manufatura enxuta. Setor automotivo. Brasil.

\begin{abstract}
The aim of this paper is to verify whether the practices of lean manufacturing are positively associated with the adoption of environmental management practices in Brazilian companies in the automotive sector to test the hypothesis that by being lean the business tends to become green. To this purpose, a framework associating these concepts with the research hypothesis was proposed. The data collected from 75 companies were analyzed using Structural Equation Modeling. The main results show that lean manufacturing (LM) is positively associated with environmental management (EM), but the explanation power of LM over EM is considered weak/moderate. In fact, being lean also means being green, but other organizational variables not considered in this study may influence the greening of organizations.
\end{abstract}

Keywords: Environmental management. Lean manufacturing. Automotive sector. Brazil.

\section{Introdução}

Vários autores (WILKINSON; HILL; GOLLAN, 2001; SARKIS, 2001; YANG; HONG; MODI, 2011) afirmam que as iniciativas de gestão ambiental nas organizações podem ter maior ou menor grau de sucesso, dependendo do apoio recebido pela área de manufatura. Segundo González-Benito (2008), quanto mais proativa a área de manufatura de uma empresa, melhor tende a ser sua gestão ambiental, sendo que o apoio da área de manufatura à gestão ambiental tende a ser mais intenso quando a empresa adota práticas de manufatura enxuta. Esse tipo de relacionamento ficou conhecido como a hipótese Lean and Green e foi analisada por diversos autores (MAXWELL et al., 1998; ROTHENBERG; PIL; MAXWELL, 2001; KING; LENOX, 2001; SIMPSON; POWER, 2005; YANG; HONG; MODI, 2011). Esses autores defendem que, de forma geral, redução de desperdícios na manufatura contribui com a gestão ambiental (SIMPSON; POWER, 2005) por meio de maior eficiência do uso dos recursos de produção (ROTHENBERG; PIL; MAXWELL, 2001) e pela adoção de práticas de limpeza e melhor organização

\footnotetext{
${ }^{1}$ Faculdade de Engenharia de Bauru, Universidade Estadual Paulista - UNESP, CEP 17033-360, Bauru, SP, Brasil, e-mail: ablsjabbour@gmail.com; prof.charbel@gmail.com

${ }^{2}$ Curso de Administração, Universidade Federal do Mato Grosso do Sul - UFMS, Rod. BR 497, Km 12, CEP 79500-000, Paranaíba, MS, Brasil, e-mail: wesley007adm@gmail.com

${ }^{3}$ Faculdade de Economia, Administração e Contabilidade de Ribeirão Preto, Universidade de São Paulo - USP, CEP 14040-905, Ribeirão Preto, SP, Brasil, e-mail: aatadrianobirigui@gmail.com
}

Recebido em 21/11/2011 — Aceito em 3/9/2012

Suporte financeiro: UNESP. 
do ambiente produtivo (KING; LENOX, 2001), o que pode gerar vantagens competitivas (YANG; HONG; MODI, 2011). Mais recentemente, Dües, Tan e Lim (2012) reafirmaram esses pressupostos.

Entretanto, principalmente à luz dos resultados de pesquisas brasileiras, pode-se dizer que há uma relativa carência de trabalhos que investigam se a hipótese de Lean and Green é válida para empresas nacionais. Dessa forma, há oportunidade de pesquisa associada à análise desse relacionamento. Idealmente, esse relacionamento deve ser inicialmente verificado no setor industrial, que é considerado por alguns pesquisadores (WOMACK; JONES; ROOS, 2004) o cenário inicial para o surgimento de práticas de gestão pioneiras: o setor automotivo. O Brasil foi escolhido como país de análise, seja pelo crescente interesse de seus pesquisadores em gestão ambiental, seja pela alta relevância do setor automotivo no PIB do país.

Assim, pode-se dizer que a questão motivadora desta pesquisa é: estariam as práticas de manufatura enxuta se relacionando positivamente com a adoção de práticas de gestão ambiental? Para responder a esta questão, os objetivos de investigação são:

- Sistematizar fundamentos conceituais sobre a relação entre gestão ambiental e manufatura enxuta;

- Propor um framework e hipótese de pesquisa para testá-la à luz de evidências empíricas por meio da Modelagem de Equações Estruturais; e

- Verificar, empiricamente, se a manufatura enxuta se relaciona positivamente com gestão ambiental.

Este artigo está organizado em cinco seções além desta introdução. Na seção 2, apresentam-se os fundamentos conceituais, oframework e a hipótese de pesquisa. Na seção 3, apresentam-se os procedimentos metodológicos. A seguir, nas seções 4 e 5, são apresentados os resultados e as discussões, e, por fim, na seção 6, apresentam-se as considerações finais.

\section{Fundamentos conceituais, framezork e hipótese de pesquisa}

\subsection{Gestão ambiental nas organizações}

Toda atividade produtiva deveria incorporar um novo modelo de gestão empresarial, que compatibiliza produção e gestão adequada dos recursos naturais (CLAVER et al., 2007). Nesse sentido, a gestão ambiental refere-se à incorporação de objetivos e estratégias ambientais aos objetivos e estratégias organizacionais (HADEN; OYLER; HUMPHREYS, 2009), além de considerar a integração das questões ambientais na produção e/ou utilização de um produto ou processo, a fim de evitar ou reduzir o impacto sobre o meio ambiente e, ao mesmo tempo, aumentar a vantagem competitiva, melhorar a imagem e participação em mercados (KASSOLIS, 2007). Jabbour, Santos e Nagano (2010) complementam tal definição indicando que a gestão ambiental deve estar baseada em uma abordagem sistêmica para a incorporação da temática ambiental em todos os níveis organizacionais.

Diversas motivações levam uma empresa a adotar práticas de gestão ambiental (BERRY; RONDINELLI, 1998). Uma delas é a pressão dos stakeholders, que, segundo González-Benito e González-Benito (2006), é o principal fator que leva as organizações a buscar uma gestão ambiental mais avançada. Além disso, uma gestão ambiental proativa pode melhorar a performance financeira da empresa (CLAVER et al., 2007; MOLINA-AZORÍN et al., 2009), incrementar sua competitividade manufatureira, promovendo redução de custos, melhoria da qualidade e geração de novos produtos e processos (YANG et al., 2010). Kassolis (2007) complementa destacando que a incorporação da gestão ambiental na empresa pode melhorar as oportunidades de mercado, reduzir custos, promover a competitividade, isentar de obrigações regulamentares, motivar os colaboradores, melhorar o desempenho da cadeia de suprimentos, fortalecer a imagem e as relações com os stakeholders e promover melhorias no desempenho ambiental.

Algumas das principais práticas de gestão ambiental são descritas no Quadro 1.

\subsection{Princípios e práticas da manufatura enxuta}

Na década de 1990, as práticas pioneiras da Toyota passam a ficar conhecidas como "manufatura enxuta" (SCHONBERGER, 2007), a partir da publicação do livro "A Máquina que Mudou o Mundo" (WOMACK; JONES; ROOS, 1990), passando a ser conhecida como uma importante técnica de gestão competitiva da produção.

Entretanto, até hoje, não há um consenso sobre a definição do termo manufatura enxuta, mesmo sendo discutido desde a década de 1950, em virtude de sua evolução a partir das incorporações e adaptações ocidentais (SHAH; WARD, 2007; PETTERSEN, 2009). Por exemplo, Womack, Jones e Roos (1990) afirmam que produção enxuta significa fazer mais com menos. Shah e Ward (2007) a classificam como um sistema sociotécnico integrado, cujo objetivo é eliminar desperdícios pela concomitante redução ou minimização da variabilidade interna, de fornecedores e de clientes. Para Scherrer-Rathje, Boyle e Deflorin (2009), produção enxuta é uma filosofia centrada em identificar e eliminar desperdícios em toda a cadeia de valor, não somente dentro da organização. Como consequência, há uma grande variedade de práticas de gestão e metas que a constituem (SHAH; WARD, 2003). 
Quadro 1. Práticas de gestão ambiental.

\begin{tabular}{|c|c|c|}
\hline Prática & Conceito & Autor \\
\hline Política ambiental & $\begin{array}{c}\text { Declaração precisa dos dirigentes empresariais sobre os } \\
\text { principais aspectos e impactos ambientais gerados. }\end{array}$ & Boiral (2006) \\
\hline Treinamento ambiental & $\begin{array}{c}\text { Treinamento ambiental para todos os funcionários tem } \\
\text { como finalidade divulgar a política ambiental e permitir } \\
\text { conscientização dos funcionários sobre os aspectos/ } \\
\text { impactos ambientais de suas atividades. }\end{array}$ & Daily e Huang (2001) \\
\hline 3Rs & $\begin{array}{c}\text { Envolvem Redução, Reuso e Reciclagem aplicados em } \\
\text { água, energia elétrica, papel e outros insumos naturais, } \\
\text { aumentando a produtividade empresarial. }\end{array}$ & Marcus e Fremeth (2009) \\
\hline Novos produtos & $\begin{array}{c}\text { Desenvolvimento de produtos com menores impactos } \\
\text { ambientais. }\end{array}$ & Sarkis (2001) \\
\hline Processo de produção & $\begin{array}{c}\text { Desenvolvimento de processo produtivo com menores } \\
\text { impactos ambientais. }\end{array}$ & Sarkis (2001) \\
\hline Seleção de fornecedores & Seleção de fornecedores com base em critérios ambientais. & Jabbour e Jabbour (2009) \\
\hline Sistemas de gestão & $\begin{array}{c}\text { Sistema de gestão ambiental, por exemplo, a ISO 14001, } \\
\text { e/ou outros. }\end{array}$ & $\begin{array}{c}\text { ABNT NBR ISO 14001/2004 } \\
\text { (ASSOCIAÇÃO..., 2004) }\end{array}$ \\
\hline Informações voluntárias & Divulgação voluntária de informações sobre o & Boiral (2006) \\
\hline
\end{tabular}

Cita-se, frequentemente, como principais objetivos e metas da produção enxuta, a redução de custo (OHNO, 1988), fabricação de produtos sem defeitos conforme a necessidade dos clientes (WOMACK; JONES; ROOS, 1990) e foco no cliente. Sendo assim, para que se possam atingir os objetivos relatados, várias práticas podem ser estabelecidas. Com base nos trabalhos de Biazzo e Panizzolo (2000), Shah e Ward (2003), Bhasin e Burcher (2006) e Pettersen (2009), busca-se sistematizar essas principais práticas e características associadas à manufatura enxuta:

- A Melhoria contínua - busca o aperfeiçoamento incremental em qualidade, custo, entrega e projeto;

- O Just in Time - busca o fluxo contínuo da produção;

- O Kanban - é um sistema de cartões para criar um fluxo puxado;

- Desenvolvimento e a colaboração de fornecedores - consistem em atividades voltadas a desenvolver relacionamento com fornecedor a fim de obter sua colaboração;

- 5S - é uma forma de gestão visual destinada a reduzir a desordem e a ineficiência no ambiente produtivo e administrativo;

- A Manutenção produtiva total - busca melhorar a confiabilidade e a capacidade das máquinas por meio de regimes periódicos de manutenção;

- A redução de lote/redução de estoque - visa a formação de pequenos lotes de produção para reduzir o estoque em processo e aumentar a variedade;
- Funcionário multifuncional/envolvimento no processo - consistem no desenvolvimento das habilidades dos funcionários e estímulo à autonomia para evitar falhas ao longo do processo; e

- Círculos de melhoria kaizen - referem-se à promoção de discussões sistemáticas entre operadores e gestores a fim de promover a melhoria incremental contínua.

Essas práticas da manufatura enxuta têm sido também empregadas para apoiar ações voltadas a iniciativas de gestão ambiental empresarial, como discutido, com mais profundidade, na próxima seção.

\subsection{Influências da manufatura enxuta na gestão ambiental empresarial}

A função produção é considerada central para a gestão ambiental empresarial (FLORIDA, 1996; JABBOUR, 2010). Assim, é importante integrar conceitos de preservação ambiental ao modelo de produção vigente na organização.

Segundo King e Lenox (2001), a lógica de organização e limpeza de práticas de produção enxuta traz o benefício de reduzir desperdícios e riscos de acidentes. Para Maxwell et al. (1998), as instalações que incorporam a produção enxuta foram dedicadas a uma filosofia de redução de desperdícios que poderiam facilmente ser estendidas para alcançar os objetivos de proteção ambiental. Vais et al. (2006) sugerem que, para se tornar enxuta e ambientalmente amigável a organização, deve se concentrar no consumo de energia e nos resíduos de materiais, que são inputs e outputs de um sistema 
de transformação. Neste contexto, associa-se que a adoção de práticas de manufatura enxuta melhora o desempenho ambiental organizacional.

Para Yang, Hong e Modi (2011), é importante que as empresas de manufatura implementem tanto as práticas de manufatura enxuta, como as de gestão ambiental, como forma de obter ecovantagem por meio de melhorias de desempenho ambiental. Como consequência, as empresas atenderão melhor os seus objetivos, em um processo sinérgico.

Algumas pesquisas relataram o modus operandi de relacionamento entre a manufatura enxuta e a gestão ambiental empresarial. Maxwell et al. (1998), Rothenberg, Pil e Maxwell (2001) e Simpson e Power (2005) enfatizam a importância do envolvimento dos funcionários, seja para intervir no processo para evitar falhas (que causam retrabalhos e uso desnecessário de recursos), ou para se comprometer e propor melhorias relativas ao melhor uso e conservação de insumos. Nesse mesmo sentido, os círculos de qualidade também são uma forma de promover esse envolvimento, uma vez que proporcionam aos funcionários treinamentos e workshops visando à motivação para participar de projetos de eficiência ambiental e de mudança de comportamento, favorecendo o consumo responsável (VAIS et al., 2006).

Vais et al. (2006) também citam o 5S e a manutenção produtiva total como práticas da produção enxuta que auxiliam a gestão ambiental. O $5 \mathrm{~S}$ proporciona a organização e a limpeza de forma a evitar a destinação incorreta de resíduos e uso equivocado de insumos. A manutenção produtiva total objetiva a periódica revisão dos equipamentos a partir de ajustes simples (limpeza, lubrificação, calibração, etc.) de forma a aumentar a vida útil do equipamento e sua eficiência (menor consumo de energia, etc.) (DONAIRE, 1999).

Segundo Pojasek (2008), as práticas da produção enxuta reforçam o alcance de alguns requisitos da Norma ISO 14001. Por exemplo, procurar a causa raiz de algum problema e, assim, aplicar ações corretivas; criar condições de prevenção de falhas (jidoka/poka yoke), proporcionando a elaboração de procedimentos e ações de emergência e melhoria contínua, a partir da análise crítica por parte da alta administração. González-Benito e González-Benito (2008) afirmam que práticas como envolvimento do funcionário, melhoria contínua e colaboração dos fornecedores são importantes e influenciam o interesse inicial pela certificação ISO 14001, enquanto que práticas como o JIT facilitam a realização da certificação.

King e Lenox (2001) e Rothenberg, Pil e Maxwell (2001) verificaram que altos níveis de prevenção da poluição ocorrem em plantas que utilizam práticas de produção enxuta, entre outras razões, por causa da redução de estoque. Outro fator de destaque segundo Simpson e Power (2005) e Corbett e Klassen (2006) é a importância da colaboração dos fornecedores no processo de melhoria ambiental, uma vez que são responsáveis pelo fornecimento de insumos que afetam diretamente a eficiência ambiental do produto final.

Em um artigo recente, Dües, Tan e Lim (2012) reafirmam a relação sinérgica e promissora entre produção enxuta e gestão ambiental, com influências positivas para a melhoria do desempenho ambiental em cadeias de suprimentos.

O Quadro 2 traz uma síntese de algumas interfaces entre diversas práticas de produção enxuta e a gestão ambiental empresarial.

No entanto, a relação entre produção enxuta e gestão ambiental é complexa e ainda não conclusiva, pois há alguns trade-offs que emergem desse processo. Por exemplo, segundo Wu e Dunn (2005), a adoção de entregas JIT acarreta o aumento de frequência de transporte que, consequentemente, afeta o aumento do tráfego rodoviário e cria demandas por novas estradas, gerando evidentes impactos ambientais. Rothenberg, Pil e Maxwell (2001) detectaram que o JIT aumenta a emissão de compostos orgânicos voláteis. King e Lenox (2001) mencionam que o aumento da eficiência em JIT requer menores lotes e mais set up produtivo, podendo gerar mais resíduos oriundos dos produtos de limpeza dos equipamentos e mais descartes de materiais de processos.

Então, é importante continuar buscando evidências empíricas para desvendar as relações entre produção enxuta e gestão ambiental, conforme objetivo deste artigo.

\subsection{Framework e hipótese de pesquisa}

$\mathrm{Na}$ Figura 1, é apresentado o framework da pesquisa com os relacionamentos entre os construtos de Manufatura Enxuta (LM, do inglês Lean Manufacturing) e de Gestão Ambiental (EM, do inglês Environmental Management). Com base nos argumentos da literatura especializada (DÜES; TAN; LIM, 2012 e outros), declara-se como hipótese de pesquisa: $\mathrm{H}_{1}$ : As práticas de manufatura enxuta (LM) se relacionam positivamente com as práticas de gestão ambiental (EM) em empresas do setor automotivo - autopeças - do Brasil.

Esse framework foi testado empiricamente, conforme os procedimentos metodológicos abaixo descritos.

\section{Metodologia}

Para responder ao objetivo proposto nesta pesquisa e para testar a hipótese estabelecida visando confirmar ou refutar o referencial teórico, adotou-se como método a pesquisa quantitativa, baseada na estratégia survey. $\mathrm{O}$ objeto de pesquisa foi o segmento de autopeças nacional, integrante do setor automotivo.

O setor automotivo brasileiro teve início na década de 1950, no governo de Juscelino Kubitschek, e atualmente movimenta diretamente 5,2\% do Produto Interno 
Quadro 2. Interfaces entre práticas de produção enxuta e apoio à gestão ambiental empresarial.

\begin{tabular}{|c|c|c|}
\hline $\begin{array}{c}\text { Práticas de produção } \\
\text { enxuta }\end{array}$ & Potencial apoio à gestão ambiental & Autor(es) \\
\hline $\begin{array}{l}\text { Envolvimento dos } \\
\text { funcionários }\end{array}$ & $\begin{array}{l}\text { O envolvimento dos funcionários de todos os } \\
\text { níveis da organização é uma motivação para a } \\
\text { melhoria da eficiência ambiental. }\end{array}$ & $\begin{array}{c}\text { Maxwell et al. (1998), } \\
\text { Rothenberg, Pil e Maxwell } \\
\text { (2001) e Simpson e Power (2005) }\end{array}$ \\
\hline Círculo kaizen & $\begin{array}{l}\text { Proporciona a formação de workshops e } \\
\text { treinamentos no intuito de mudar a atitude das } \\
\text { pessoas para estimular o comportamento voltado } \\
\text { à limpeza do ambiente de produção, à redução } \\
\text { do consumo de insumos, ao reuso de recursos e à } \\
\text { reciclagem de materiais. }\end{array}$ & $\begin{array}{l}\text { Maxwell et al. (1998), } \\
\text { Rothenberg, Pil e Maxwell } \\
\text { (2001) e Vais et al. (2006) }\end{array}$ \\
\hline $5 \mathrm{~S}$ & $\begin{array}{l}\text { Manutenção da limpeza e da organização do } \\
\text { ambiente de trabalho de forma a reduzir o } \\
\text { consumo de insumos e descartar adequadamente } \\
\text { rejeitos de produção. }\end{array}$ & Vais et al. (2006) \\
\hline Manutenção produtiva total & $\begin{array}{c}\text { Manutenção preventiva e proativa nos } \\
\text { equipamentos de forma a aumentar a vida útil e } \\
\text { evitar falhas de processos que gerem refugos e } \\
\text { retrabalhos. }\end{array}$ & Donaire (1999) e Vais et al. (2006) \\
\hline Melhoria contínua & $\begin{array}{l}\text { Reforça e suporta a criação e manutenção de um } \\
\text { sistema de gestão ambiental. }\end{array}$ & $\begin{array}{c}\text { Pojasek (2008) e González-Benito } \\
\text { e González-Benito (2008) }\end{array}$ \\
\hline Redução de estoque & $\begin{array}{c}\text { Facilita a identificação de falhas no processo } \\
\text { e com isso evita gerar consumo excessivo e } \\
\text { geração de desperdícios. }\end{array}$ & $\begin{array}{l}\text { Rothenberg et al. (2001) e King e } \\
\text { Lenox (2001) }\end{array}$ \\
\hline Colaboração do fornecedor & $\begin{array}{l}\text { A responsabilidade ambiental é estendida aos } \\
\text { fornecedores. }\end{array}$ & $\begin{array}{l}\text { Simpson e Power (2005), Corbett e } \\
\text { Klassen (2006) e González-Benito } \\
\text { e González-Benito (2008) }\end{array}$ \\
\hline
\end{tabular}

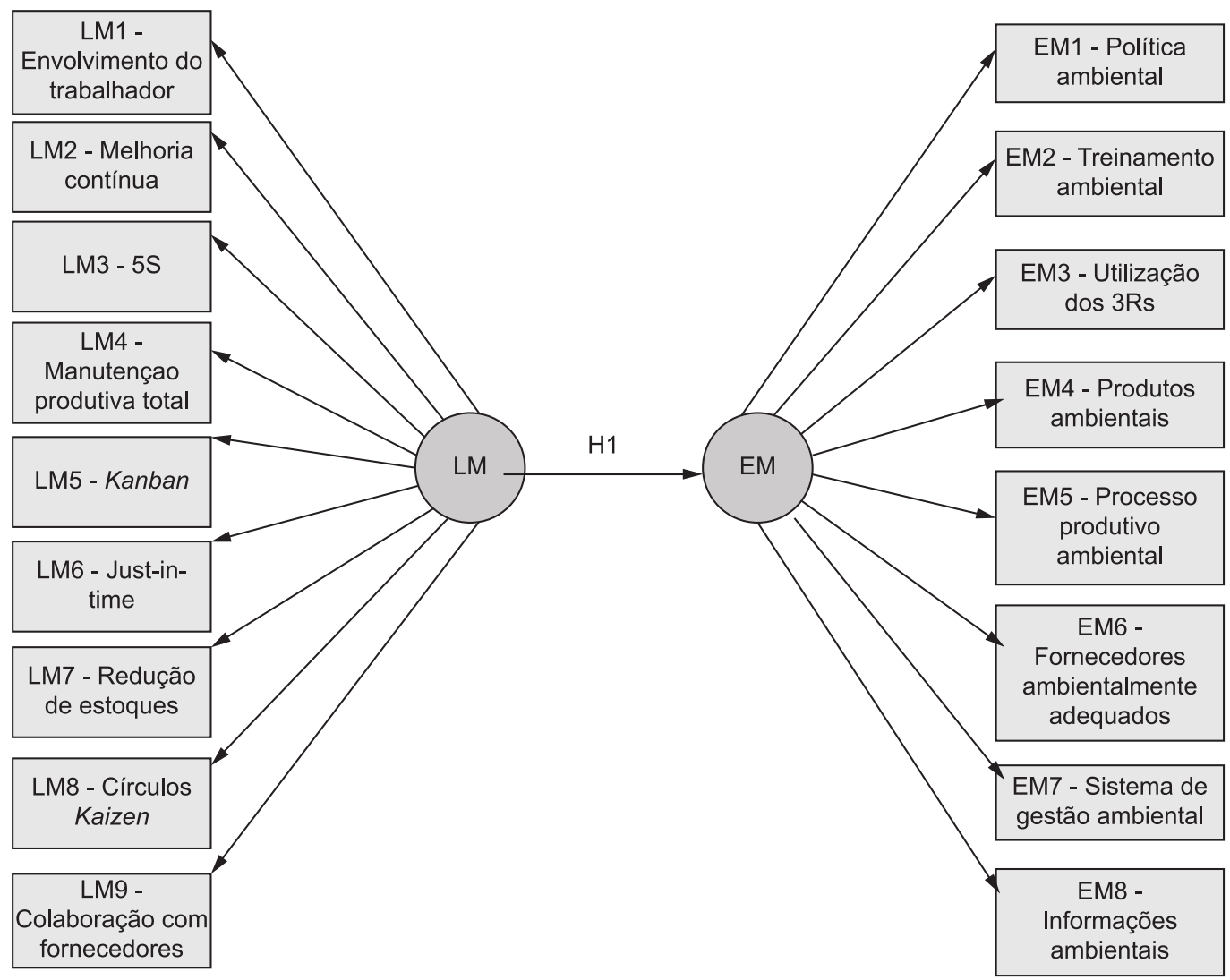

Figura 1. Framework relacional da hipótese da pesquisa. 
Bruto Brasileiro (PIB) (ASSOCIAÇÃO..., 2011). Destaca-se que o segmento de autopeças no Brasil contribui com o crescimento do setor automotivo, principalmente, contribuindo com inovações em peças e matérias-primas.

Para a coleta de dados, foi planejado um instrumento do tipo questionário estruturado, autoadministrado pelos respondentes, sobre os conceitos previamente revisados nas seções 2.1, 2.2 e 2.3, elaborado segundo as recomendações contidas em Synodinos (2003).

O questionário elaborado possui, além de informações sobre a caracterização das empresas respondentes, dois blocos de assertivas, um para o construto "Gestão

Tabela 1. Resultado da Análise de Componentes Principais para LM.

\begin{tabular}{ccc}
\hline Variáveis & Carga & Comunalidades \\
\hline LM1 & 0,79 & 0,63 \\
LM2 & 0,84 & 0,71 \\
LM3 & 0,81 & 0,65 \\
LM4 & 0,81 & 0,66 \\
LM5 & 0,74 & 0,54 \\
LM6 & 0,81 & 0,65 \\
LM7 & 0,82 & 0,67 \\
LM8 & 0,82 & 0,68 \\
LM9 & 0,75 & 0,57 \\
\hline
\end{tabular}

Tabela 2. Média e desvio padrão das variáveis do Construto LM.

\begin{tabular}{ccc}
\hline Variáveis & Média & Desvio padrão \\
\hline LM1 & 3,69 & 1,12 \\
LM2 & 3,86 & 1,05 \\
LM3 & 3,78 & 1,18 \\
LM4 & 3,20 & 1,27 \\
LM5 & 2,90 & 1,41 \\
LM6 & 3,04 & 1,42 \\
LM7 & 3,52 & 1,01 \\
LM8 & 3,20 & 1,37 \\
LM9 & 3,17 & 1,18 \\
\hline
\end{tabular}

Ambiental (EM)" e outro para "Manufatura Enxuta (LM)". No total, o questionário (Apêndice 1) possuía oito assertivas sobre gestão ambiental (uma para cada prática ambiental) e nove assertivas para "Manufatura Enxuta". A primeira versão do questionário foi submetida à validação de conteúdo, por meio da análise de cinco pesquisadores na área, além de adequação aos pressupostos conceituais. O questionário, em sua versão final, foi hospedado em um ambiente virtual especialmente elaborado para esta pesquisa. Adotou-se uma escala Likert de 5 pontos, em que 1 representa "não implementado" e 5 representa "totalmente implementado", conforme o Apêndice 1 deste artigo.

Os dados desta pesquisa foram coletados entre os meses de outubro de 2010 e março de 2011. Primeiramente, foram coletados endereços de e-mail e informações telefônicas, junto ao Sindicato Nacional da Indústria de Componentes para Veículos Automotores - SINDIPEÇAS, de 654 empresas do setor automotivo (segmento de autopeças) localizadas no Brasil. Foram enviados e-mails para esse conjunto de empresas, contendo uma breve explicação sobre a pesquisa e um convite para participação direcionado ao gestor de produção. No e-mail, havia um link para direcionar o respondente-alvo diretamente para o questionário hospedado no ambiente virtual da pesquisa. Também foram realizadas ligações telefônicas direcionadas para o aumento do percentual de retorno de questionários válidos, em que se buscou contatar o funcionário responsável pela área de Produção/Operações da empresa. Assim, foram 72 questionários coletados por meio do site da pesquisa e 4 questionários coletados por meios alternativos, conforme solicitado pelos respectivos respondentes. Foram, assim, obtidos 76 questionários, dos quais 1 foi desconsiderado por estar incompleto. Totalizou-se uma taxa de retorno de 11,11\%, (75 questionários válidos), valor considerado adequado frente aos percentuais indicados por Synodinos (2003). Cada questionário preenchido alimentou automaticamente uma planilha de dados para posterior processamento estatístico.

Tabela 3. Correlação de Pearson para as variáveis do Construto LM.

\begin{tabular}{cccccccccc}
\hline & LM1 & LM2 & LM3 & LM4 & LM5 & LM6 & LM7 & LM8 & LM9 \\
\hline LM1 & 1 & & & & & & & & \\
LM2 & $0,737^{*}$ & 1 & & & & & & & \\
LM3 & $0,637^{*}$ & $0,708^{*}$ & 1 & & & & & & \\
LM4 & $0,627^{*}$ & $0,653^{*}$ & $0,671^{*}$ & 1 & & & & & \\
LM5 & $0,499^{*}$ & $0,479^{*}$ & $0,518^{*}$ & $0,535^{*}$ & 1 & & & & \\
LM6 & $0,512^{*}$ & $0,568^{*}$ & $0,546^{*}$ & $0,657^{*}$ & $0,771^{*}$ & 1 & & & \\
LM7 & $0,600^{*}$ & $0,643^{*}$ & $0,528^{*}$ & $0,534^{*}$ & $0,615^{*}$ & $0,701^{*}$ & 1 & & \\
LM8 & $0,598^{*}$ & $0,688^{*}$ & $0,638^{*}$ & $0,579^{*}$ & $0,530^{*}$ & $0,581^{*}$ & $0,677^{*}$ & 1 & \\
LM9 & $0,524^{*}$ & $0,567^{*}$ & $0,590^{*}$ & $0,592^{*}$ & $0,419^{*}$ & $0,497^{*}$ & $0,627^{*}$ & $0,647^{*}$ & 1 \\
\hline
\end{tabular}

$*$ p value $<0,05$. 
O framework de pesquisa (apresentado na Seção 2.4) guiou o processo de análise de dados, que envolveu o uso de procedimentos estatísticos com o suporte de planilhas de dados do Software Statistical Package for Social Sciences da IBM (Versão 19.0) e do Software Smart PLS 2.0. A Seção 4 apresenta, detalhadamente, os procedimentos estatísticos associados a cada um dos resultados obtidos. Na Seção 5, de Discussões, é apresentada a validade ou rejeição da hipótese desta pesquisa e suas implicações.

O teste do framework e da hipótese de pesquisa seguiu os procedimentos metodológicos descritos abaixo.

\section{Resultados}

A redução das variáveis, tanto do construto Manufatura Enxuta (LM) quanto para o construto

Tabela 4. Resultado da Análise de Componentes Principais para EM.

\begin{tabular}{ccc}
\hline Variáveis & Carga & Comunalidades \\
\hline EM1 & 0,900 & 0,811 \\
EM2 & 0,936 & 0,875 \\
EM3 & 0,874 & 0,764 \\
EM4 & 0,818 & 0,669 \\
EM5 & 0,850 & 0,723 \\
EM6 & 0,808 & 0,653 \\
EM7 & 0,875 & 0,766 \\
EM8 & 0,831 & 0,690 \\
\hline
\end{tabular}

Tabela 5. Média e desvio padrão das variáveis do Construto EM.

\begin{tabular}{ccc}
\hline Variáveis & Média & Desvio Padrão \\
\hline EM1 & 3,24 & 1,71 \\
EM2 & 3,08 & 1,68 \\
EM3 & 3,02 & 1,48 \\
EM4 & 2,96 & 1,47 \\
EM5 & 3,17 & 1,39 \\
EM6 & 2,57 & 1,41 \\
EM7 & 3,04 & 1,81 \\
EM8 & 2,65 & 1,58 \\
\hline
\end{tabular}

Gestão Ambiental (EM), foi realizada usando a Análise de Componentes Principais, pelo método varimax. Esse procedimento foi realizado independentemente para cada um dos construtos. Nesse processo, os principais indicadores de qualidade estatística foram:

- Adequação da amostra para cada fator individual pelo teste KMO (Kaiser-Meyer-Olkin);

- Cálculo do Alfa de Cronbach para cada fator;

- Teste Bartlett de Esfericidade;

- Diagonal principal da Matriz Anti-Imagem;

- Comunalidades das variáveis;

- O Eigenvalue para cada fator, no qual foram extraídos os fatores com valores iguais ou superiores a 1,0 ; e

- A variância acumulada explicada.

A fim de refinar os resultados, a Análise de Componentes Principais passou a revelar apenas cargas de variáveis acima de 0,6, fatores com Eingenvalues maior que 1 e coeficientes da diagonal da matriz Anti-Imagem maior do que 0,6. Verificou-se se a comunalidade das variáveis é igual ou superior a 0,5 para cada variável (HAIR JUNIOR et al., 2005). Em relação ao Construto Manufatura Enxuta (LM), apenas um fator foi formado, explicando uma variância acumulada aproximada de 64,27\%, com Eigenvalue de 5,78 e valores adequados na diagonal principal da Matriz Anti-Imagem $(0,917 ; 0,904 ; 0,927 ; 0,903$; $0,867 ; 0,841 ; 0,891 ; 0,943 ; 0,908)$. O teste KMO, que verifica a adequação da amostra, foi de 0,900, considerado adequado, assim como o valor obtido com o teste Bartlett de Esfericidade (460,202, com adequado nível de significância) e pelo Alfa de Conbrach $(0,927)$. Todas as variáveis do Construto LM apresentaram valores satisfatórios (Tabela 1).

Depois do refinamento do construto Manufatura Enxuta (LM), conclui-se que a variável LM2 - Busca Sistemática pela Melhoria Contínua - foi aquela que obteve maior média entre as práticas de LM (Tabela 2).

Realizou-se também o teste de coeficientes de correlação de Pearson (Tabela 3), em que se verificou que todas as variáveis LM1-LM9 apresentam

Tabela 6. Correlação de Pearson para as variáveis do Construto EM.

\begin{tabular}{ccccccccc}
\hline & EM1 & EM2 & EM3 & EM4 & EM5 & EM6 & EM7 & EM8 \\
\hline EM1 & 1 & & & & & & & \\
EM2 & $0,939^{*}$ & 1 & & & & & & \\
EM3 & $0,717^{*}$ & $0,781^{*}$ & 1 & & & & & \\
EM4 & $0,624^{*}$ & $0,660^{*}$ & $0,746^{*}$ & 1 & & & & \\
EM5 & $0,687^{*}$ & $0,689^{*}$ & $0,783^{*}$ & $0,836^{*}$ & 1 & & & \\
EM6 & $0,655^{*}$ & $0,678^{*}$ & $0,653^{*}$ & $0,645^{*}$ & $0,659^{*}$ & 1 & & \\
EM7 & $0,854^{*}$ & $0,899^{*}$ & $0,676^{*}$ & $0,608^{*}$ & $0,605^{*}$ & $0,628^{*}$ & 1 & \\
EM8 & $0,700^{*}$ & $0,773^{*}$ & $0,666^{*}$ & $0,548^{*}$ & $0,623^{*}$ & $0,684^{*}$ & $0,737^{*}$ & 1 \\
\hline
\end{tabular}

$* \mathrm{p}$ value $<0,05$. 
correlações significativas, com destaque para a relação entre LM5 (Kanban) e LM6 (Just-in-Time).

Em relação ao Construto Gestão Ambiental (EM), apenas um fator foi formado, explicando uma variância acumulada aproximada de 74,38\%, com Eigenvalue de 5,95 e valores adequados na diagonal principal da Matriz Anti-Imagem $(0,848 ; 0,821 ; 0,925 ; 0,863$; $0,852 ; 0,951 ; 0,930 ; 0,908)$. O teste KMO, que verifica a adequação da amostra, foi de 0,882 , considerado adequado, assim como o valor obtido com o teste Bartlett de Esfericidade (636,937, com adequado nível de significância) e pelo Alfa de Conbrach $(0,949)$. Todas as variáveis do Construto EM apresentaram valores satisfatórios (Tabela 4).

Depois do refinamento do Construto Gestão Ambiental (EM) acima relatado, percebe-se que a variável EM1 - Política Ambiental - foi aquela que obteve maior média entre as práticas de gestão ambiental (Tabela 5).

Realizou-se também o teste de coeficientes de correlação de Pearson (Tabela 6), no qual se verificou que todas as variáveis EM1-EM8 apresentam correlações significativas, com destaque para a relação entre EM1 (Política Ambiental) e EM2 (treinamento ambiental).

Em seguida, Structural Equation Modeling - Partial Least Squares (SEM-PLS) foi utilizada. A Modelagem de Equações Estruturais por meio de PLS é considerada uma análise multivariada de segunda geração. Ela é especialmente útil quando se trabalha com teoria complexa (relacionando conceitos tradicionais, como Manufatura Enxuta e Gestão Ambiental) e/ou em estágios iniciais de desenvolvimento. Um modelo (Figura 2) foi criado contendo os construtos obtidos a partir da Análise de Componentes Principais, como explicado acima. As análises foram realizadas usando o software SmartPLS 2.03 (SOSIK; KAHAI; PIOVOSO, 2009).

Indicadores de boa qualidade para o modelo proposto foram alcançados em termos de variância média extraída (validade convergente), confiabilidade composta, alfa de Cronbach e comunalidades, tanto para o construto LM, quanto para EM. Para checar a confiabilidade (que apura a precisão com que o construto mede exatamente aquilo que realmente deveria medir) e validade satisfatórias (que testa a relação de uma variável com outra variável de um mesmo construto), o valor de Confiabilidade deve ser superior a 0,7 , enquanto o valor da Validade deve ser superior a 0,5. A Confiabilidade de construto foi avaliada utilizando Confiabilidade Composta. Já Validade foi analisada pela Variância Média Extraída. A Tabela 7 mostra que todos os valores de Confiabilidade Composta são superiores a 0,7 e todos os valores de Variância Média Extraídos são superiores a 0,5 (FOLTZ, 2008). Os Coeficientes de Alfa de Cronbach e as comunalidades também são considerados adequados.

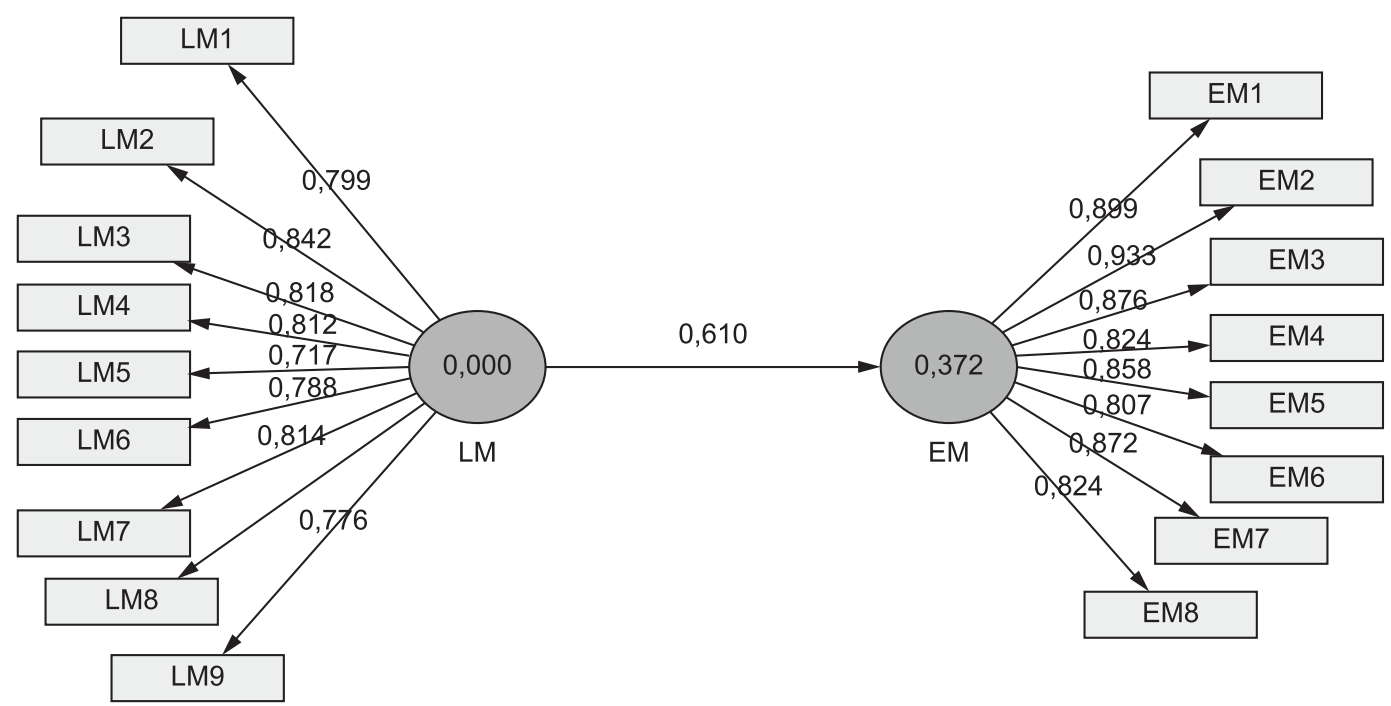

Figura 2. Modelo Estrutural.

Tabela 7. Valores de confiabilidade e de validade do Modelo Estrutural.

\begin{tabular}{cccccc}
\hline Construtos & $\begin{array}{c}\text { Variância Média } \\
\text { Extraída (AVE) }\end{array}$ & $\begin{array}{c}\text { Confiabilidade } \\
\text { composta }\end{array}$ & $\boldsymbol{R}$ Square & $\begin{array}{c}\text { Alfa de } \\
\text { Cronbach }\end{array}$ & Comunalidade \\
\hline EM & 0,74 & 0,95 & 0,37 & 0,95 & 0,74 \\
LM & 0,64 & 0,94 & - & 0,93 & 0,64 \\
\hline
\end{tabular}


Outra medida importante para avaliar o modelo estrutural é a validade discriminante, verificando se as variáveis realmente possuem cargas mais elevadas em seus fatores de origem. Essa análise obteve resultados adequados (Tabela 8).

Com vistas ao teste de robustez do modelo, um bootstrap de 1.000 subamostras foi utilizado para estimar a significância estatística das relações entre as variáveis propostas e os construtos (Figura 3).

Tabela 8. Cargas cruzadas para avaliação da validade discriminante.

\begin{tabular}{lll}
\hline & EM & LM \\
\hline EM 1 & $\mathbf{0 , 8 9}$ & 0,54 \\
EM 2 & $\mathbf{0 , 9 3}$ & 0,54 \\
EM 3 & $\mathbf{0 , 8 7}$ & 0,53 \\
EM 4 & $\mathbf{0 , 8 2}$ & 0,50 \\
EM 5 & $\mathbf{0 , 8 5}$ & 0,58 \\
EM 6 & $\mathbf{0 , 8 0}$ & 0,48 \\
EM 7 & $\mathbf{0 , 8 7}$ & 0,54 \\
EM 8 & $\mathbf{0 , 8 2}$ & 0,44 \\
LM1 & 0,50 & $\mathbf{0 , 7 9}$ \\
LM2 & 0,46 & $\mathbf{0 , 8 4}$ \\
LM3 & 0,52 & $\mathbf{0 , 8 1}$ \\
LM4 & 0,48 & $\mathbf{0 , 8 1}$ \\
LM5 & 0,35 & $\mathbf{0 , 7 1}$ \\
LM6 & 0,41 & $\mathbf{0 , 7 8}$ \\
LM7 & 0,43 & $\mathbf{0 , 8 1}$ \\
LM8 & 0,56 & $\mathbf{0 , 8 3}$ \\
LM9 & 0,57 & $\mathbf{0 , 7 7}$ \\
\hline
\end{tabular}

Todas as relações do modelo são estatisticamente válidas ao nível de significância ( $p$ value) menor ou igual a 0,01 , conforme a Tabela 9 .

A partir desses resultados, pode-se concluir que, de fato, a manufatura enxuta (LM) vem se relacionando positivamente com a gestão ambiental (EM) nas empresas analisadas. Essa relação pode ser considerada positiva, pois a carga entre LM e EM é de aproximadamente 0,6 e estatisticamente válida pelo $p$ value obtido. $\mathrm{O}$ valor de determinação de LM em EM foi de 0,372, podendo ser considerado entre fraco e moderado, segundo Hair Junior, Ringle e Sarstedt (2011).

\section{Discussões}

Percebe-se que o construto Manufatura Enxuta (LM) teve todas as suas variáveis validadas. Entre todas as práticas, a "busca sistemática pela melhoria contínua" foi aquela que obteve maior média de implantação e foi também a variável mais importante no teste do modelo para o construto Manufatura Enxuta, confirmando argumentos da literatura especializada (BHUIYAN; BAGHEL, 2005). Em termos de correlação, verificou-se interdependência entre todas as variáveis de Manufatura Enxuta, com destaque para a relação entre LM5 (Kanban) e LM6 (Just in Time). Essa correlação pode ser explicada pela importância dos sistemas kanban para a implementação do Just-in-Time.

Pode-se verificar que a gestão ambiental nas empresas analisadas tende a ser composta pela totalidade das práticas aqui consideradas, confirmando

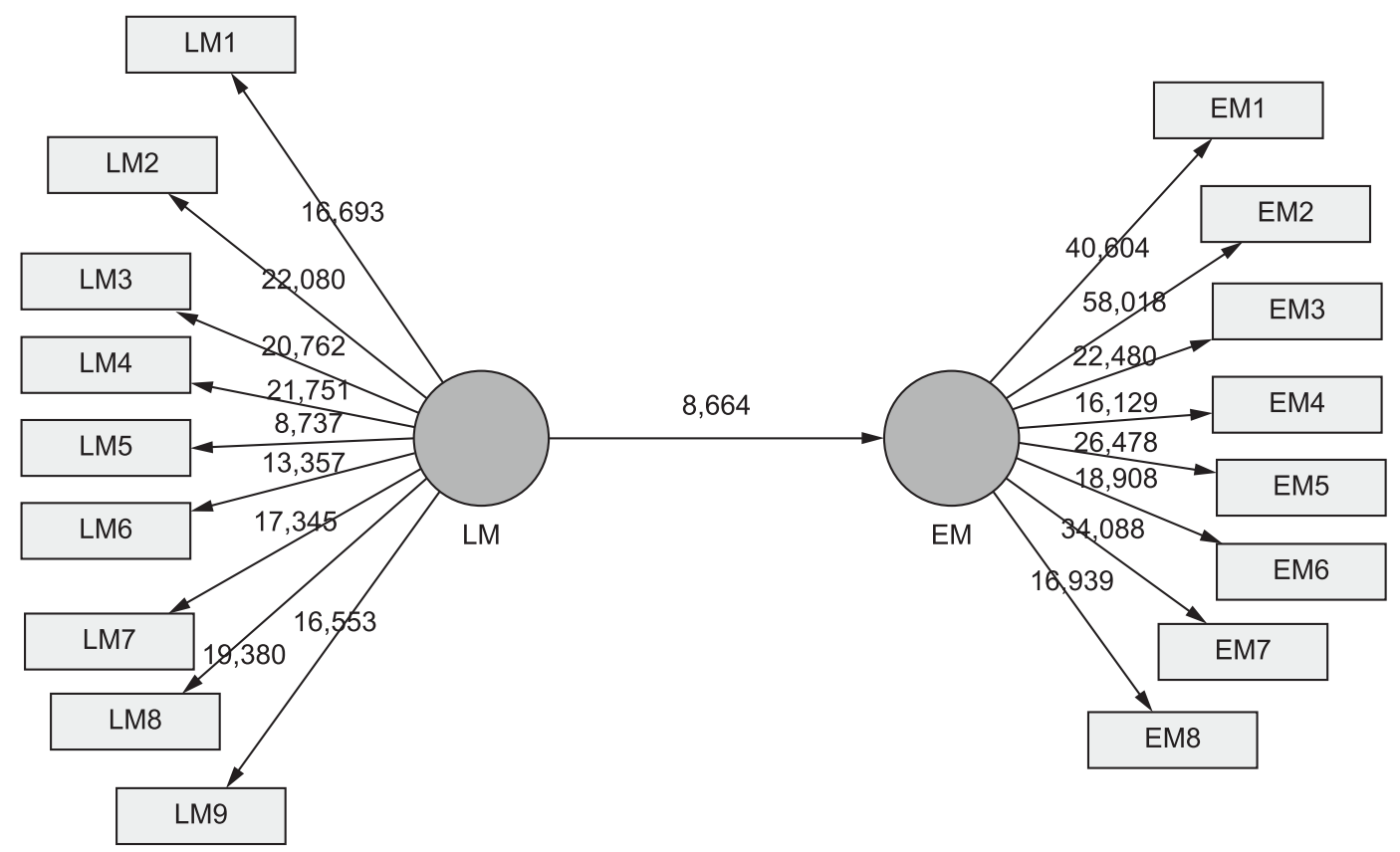

Figura 3. Bootstrapping de 1.000 subamostragens. 
Tabela 9. Significância dos Coeficientes dos relacionamentos do modelo.

\begin{tabular}{cccc}
\hline Relacionamentos & Coeficiente & Teste t & Significância \\
\hline LM $\rightarrow$ EM & 0,61 & 8,90 & $*$ \\
EM1 $\leftarrow$ EM & 0,89 & 40,44 & $*$ \\
EM2 $\leftarrow$ EM & 0,93 & 60,10 & $*$ \\
EM3 $\leftarrow$ EM & 0,87 & 21,94 & $*$ \\
EM4 $\leftarrow$ EM & 0,82 & 16,00 & $*$ \\
EM5 $\leftarrow$ EM & 0,85 & 25,65 & $*$ \\
EM6 $\leftarrow$ EM & 0,80 & 18,75 & $*$ \\
EM7 $\leftarrow$ EM & 0,87 & 33,77 & $*$ \\
EM8 $\leftarrow$ EM & 0,82 & 17,35 & $*$ \\
LM1 $\leftarrow$ LM & 0,79 & 16,73 & $*$ \\
LM2 $\leftarrow$ LM & 0,84 & 22,08 & $*$ \\
LM3 $\leftarrow$ LM & 0,81 & 20,51 & $*$ \\
LM4 $\leftarrow$ LM & 0,81 & 21,71 & $*$ \\
LM5 $\leftarrow$ LM & 0,71 & 8,81 & $*$ \\
LM6 $\leftarrow$ LM & 0,78 & 14,01 & $*$ \\
LM7 $\leftarrow$ LM & 0,81 & 17,47 & $*$ \\
LM8 $\leftarrow$ LM & 0,83 & 19,68 & $*$ \\
LM9 $\leftarrow$ LM & 0,77 & 16,05 & $*$ \\
\hline
\end{tabular}

$*$ p value $<0,01$.

as indicações de González-Benito e González-Benito (2006) sobre a implementação da gestão ambiental por meio de um composto de práticas. Houve destaque para a prática de "política ambiental", que apresentou maior média, e para a variável "treinamento ambiental", que apresentou maior coeficiente de correlação com o construto gestão ambiental, cuja importância foi destacada por diversos autores (GOVINDARAJULU; DAILY, 2004; DAILY; HUANG, 2001; SARKIS; GONZALEZ-TORRE; ADENSO-DIAZ, 2010).

A hipótese $\mathrm{H}_{1}$, de que a manufatura enxuta se relaciona positivamente com a gestão ambiental das empresas da amostra, foi validada. Essa validação é corroborada por argumentos clássicos da literatura sobre os benefícios da manufatura enxuta para a gestão ambiental, que ficaram conhecidos como paradigma lean and green. Assim, espera-se um efeito sinérgico entre manufatura enxuta e gestão ambiental (DÜES; TAN; LIM, 2012). Um resultado surpreendente foi a relação positiva entre a variável LM6 - Just in Time no construto LM relacionado a EM (KING; LENOX, 2001). Embora a literatura afirme que o Just in Time seja geralmente pouco benéfico à gestão ambiental, essa percepção não foi verificada junto aos respondentes desta pesquisa.

\section{Conclusões}

Esta pesquisa teve como objetivo verificar se a manufatura enxuta tende a se relacionar positivamente com a adoção de práticas de gestão ambiental em empresas do setor automotivo no Brasil. O objetivo proposto foi alcançado por meio de um teste do framework e da hipótese de pesquisa com base em 75 empresas brasileiras. Os principais resultados obtidos foram:

- Todas as práticas de manufatura enxuta tendem a ser adotadas nas empresas analisadas, com destaque para a prática de busca da melhoria contínua (BHUIYAN; BAGHEL, 2005);

- Todas as práticas de gestão ambiental tendem a ser adotadas nas empresas analisadas, com destaque para as práticas de política ambiental e de treinamento ambiental; e

- As práticas de manufatura enxuta tendem a suportar, de forma positiva e estatisticamente válida, a gestão ambiental nas empresas analisadas, mas o poder de determinação de LM em EM pode ser considerado como fraco-moderado, tendo em vista o $\mathrm{R}^{2}$ obtido.

Esses resultados confirmam alguns pressupostos da literatura sobre lean and green. Por exemplo, verifica-se que, pelo relacionamento estatístico evidenciado, parece haver um relacionamento sistêmico entre manufatura enxuta e gestão ambiental nas empresas, conforme sugere Dües, Tan e Lim (2012). Entretanto, eventuais correlações negativas e inversamente proporcionais entre Just in Time e gestão ambiental não foram verificadas nesta pesquisa, contrariando as indicações de King e Lenox (2001) e de Rothenberg, Pil e Maxwell (2001). Por fim, as práticas de gestão ambiental mais relevantes (política ambiental e treinamento ambiental) fazem parte do conjunto de práticas ambientais organizacionais e de planejamento (GONZÁLEZ-BENITO; GONZÁLEZBENITO, 2006) e vêm sendo ressaltadas como relevantes para empresas que buscam incremento em sua sustentabilidade (DAILY; HUANG, 2001). Essas duas práticas fazem parte do lado soft e intangível da gestão ambiental que vem recebendo crescente atenção dos pesquisadores em gestão (GOVINDARAJULU; DAILY, 2004).

Os resultados desta pesquisa possuem implicações gerenciais. Primeiramente, chama-se a atenção para o comportamento sinérgico entre as práticas de LM e de EM. Dessa forma, gestores interessados em melhorar a performance ambiental de suas empresas devem investir não só em práticas ambientais, mas também em práticas de manufatura enxuta; precisam também possuir uma perspectiva interdisciplinar e para além das restrições das áreas funcionais organizacionais para viabilizar a implementação de práticas ambientais. Por fim, cabe destacar que o poder explicativo de LM em EM foi considerado fraco-moderado. De tal forma, outras variáveis organizacionais não contempladas neste estudo podem estar determinando o desempenho da amostra de empresas em EM. Essas variáveis, 
seu comportamento e características precisam ser analisados em pesquisas futuras. Por exemplo, as variáveis "cultura organizacional” ou "estrutura organizacional", entre muitas outras possíveis, podem estar influenciando, em alguma medida, o desempenho das empresas da amostra em gestão ambiental.

Esses resultados podem ser úteis para os profissionais dedicados ao ensino de disciplinas sobre gestão ambiental, manufatura enxuta ou produção sustentável. Podem também ser úteis para disciplinas sobre Doing Business in Brazil.

\section{Referências}

ASSOCIAÇÃO BRASILEIRA DE NORMAS TÉCNICAS - ABNT. NBR ISO 14001/2004: Associação Brasileira de Normas Técnicas. Sistema de Gestão Ambiental. Especificação e diretrizes para uso. Rio de Janeiro. ABNT, 2004.

ASSOCIAÇÃO NACIONAL DOS FABRICANTES DE VEÍCULOS AUTOMOTORES - ANFAVEA. Disponível em: <www.anfavea.org.br>. Acesso em: jun. 2011.

BHASIN, S.; BURCHER, P. Lean viewed as a philosophy. Journal of Manufacturing Technology Management, v. 17, n. 1, p. 56-72, 2006. http://dx.doi. org/10.1108/17410380610639506

BERRY, M. A.; RONDINELLI, D. A. Proactive environmental management: A new industrial revolution. The Academy of Management Executive, v. 12, n. 2, p. 38-50, 1998.

BHUIYAN, N.; BAGHEL, A. An overview of continuous improvement: from the past to the present. Management Decision, v. 43, n. 5, p. 761-771, 2005. http://dx.doi. org/10.1108/00251740510597761

BIAZZO, S.; PANIZZOLO, R. The assessment of work organization in lean production: the relevance of the worker's perspective. Integrated Manufacturing Systems, v. 11, n. 1, p. 6-15, 2000. http://dx.doi. org/10.1108/09576060010303622

BOIRAL, O. Global warming: should companies adopt a proactive strategy? Long Range Planning, v. 39, p. 315-330, 2006. http://dx.doi.org/10.1016/j. lrp.2006.07.002

CLAVER, E. et al. Environmental management and firm performance: A case study. Journal of Environmental Management, v. 84, n. 4, p. 606-619, 2007. http:// dx.doi.org/10.1016/j.jenvman.2006.09.012

CORBETT, C. J.; KLASSEN, R. D. Extending the horizons: environmental excellence as key to improving operations. Manufacturing \& Service Operations Management, v. 8, n. 1, p. 5-22, 2006. http://dx.doi.org/10.1287/ msom.1060.0095

DAILY B.; HUANG, S. Achieving sustainability through attention to human resource factors in environmental management. International Journal of Operations \& Production Management, v. 21, n. 12, p. 1539-1552, 2001. http://dx.doi.org/10.1108/01443570110410892

DONAIRE, D. Gestão ambiental na empresa. São Paulo: Atlas, 1999.
DÜES, C. M.; TAN, K. H.; LIM, M. Green as the new lean: how to use lean practices as a catalyst to greening your supply chain. Journal of Cleaner Production, 2012. In press. http://dx.doi.org/10.1016/j.jclepro.2011.12.023

FLORIDA, R. Lean and green: the move to environmentally conscious manufacturing. California Management Review, v. 39, n. 1, p. 80-105, 1996.

FOLTZ, C. B. Why users (fail to) read computer usage policies. Industrial Management \& Data Systems, v. 108, n. 6, 701-12, 2008. http://dx.doi. org/10.1108/02635570810883969

GONZÁLEZ-BENITO, J. The effect of manufacturing pro-activity on environmental management: an exploratory analysis. International Journal of Production Research, v. 46, n. 24, p. 7017-7038, 2008. http://dx.doi.org/10.1080/00207540701474021

GONZÁLEZ-BENITO, J.; GONZÁLEZ-BENITO, O. Environmental proactivity and business performance: an empirical analysis. Omega: The International Journal of Management Science, v. 33, p. 1-15, 2006.

GONZÁLEZ-BENITO, J.; GONZÁLEZ-BENITO, O. Operations management practices linked to the adoption of ISO 14001: an empirical analysis of Spanish manufacturers. International Journal of Production Economics, v. 113, p. 60-73, 2008. http://dx.doi. org/10.1016/j.ijpe.2007.02.051

GOVINDARAJULU, N.; DAILY, B. F. Motivating employees for environmental improvement. Industrial Management \& Data Systems, v. 104, n. 4, p. 364-372, 2004. http:// dx.doi.org/10.1108/02635570410530775

HADEN, S. S. P.; OYLER, J. D.; HUMPHREYS, J. H. Historical, practical, and theoretical perspectives on green management: An exploratory analysis. Management Decision, v. 47, n. 7, p. 1041-55, 2009. http://dx.doi. org/10.1108/00251740910978287

HAIR JUNIOR, J. F. et al. Fundamentos de métodos de pesquisa em administração. Porto Alegre: Bookman, 2005.

HAIR JUNIOR, J. F.; RINGLE, C. M.; SARSTEDT, M. PLS-SEM: Indeed a silver bullet. Journal of Marketing Theory and Practice, v. 19, n. 2, p. 139-151, 2011. http://dx.doi.org/10.2753/MTP1069-6679190202

JABBOUR, A. B. L. S.; JABBOUR, C. J. C. Are supplier selection criteria going green? Case studies of company in Brazil. Industrial Management \& Data Systems, v. 109, n. 4, p. 477-495, 2009. http://dx.doi. org/10.1108/02635570910948623

JABBOUR, C. J. C. In the eye of the storm: exploring the introductions of environmental issues in the production function in Brazilian companies. International Journal of Production Research, v. 48, n. 21, p. 6315-6339, 2010. http://dx.doi.org/10.1080/00207540802425401

JABBOUR, C. J. C.; SANTOS, F. C. A.; NAGANO, M. S. Contributions of HRM throughout the stages of environmental management: methodological triangulation applied to companies in Brazil. International Journal of Human Resource Management, v. 21, n. 7, p. 10491089, 2010. http://dx.doi.org/10.1080/09585191003783512

KASSOLIS, M. G. The diffusion of environmental management in Greece through rationalist approaches: driver or product of globalisation? Journal of Cleaner 
Production, v. 15, n. 18, p. 1886-1893, 2007. http:// dx.doi.org/10.1016/j.jclepro.2007.02.006

KING, A. A.; LENOX, M. J. Lean and green? an empirical examination of the relationship between lean production and environmental performance. Production and Operations Management, v. 10, n. 3, p. 244-256, 2001. http://dx.doi.org/10.1111/j.1937-5956.2001.tb00373.x

MARCUS, A.; FREMETH, A. Green Management Matters Regardless. Academy of Management Perspectives, v. 23, n. 4, p. 17-26, 2009. http://dx.doi.org/10.5465/ AMP.2009.43479261

MAXWELL, J. et al. Case study: Honda of American manufacturing, inc.: can lean production practices increase environmental performance? Environmental Quality Management, v. 8, n. 1, p. 53-61, 1998. http:// dx.doi.org/10.1002/tqem.3310080107

MOLINA-AZORIN, J. F. et al. Green management and financial performance: a literature review. Management Decision, v. 47, n. 7, p. 1080-1100, 2009. http://dx.doi. org/10.5465/AMP.2009.43479261

OHNO, T. Toyota production system: beyond large scale production. Cambrigde: Productivity Press, 1988.

PETTERSEN, J. Defining lean production: some conceptual and practical issues. The TQM Journal, v. 21, n. 2, p. 127-142, 2009. http://dx.doi. org/10.1108/17542730910938137

POJASEK, R. B. Framing your lean-to-green effort. Environmental Quality Management, v. 18, p. 85-93, 2008. http://dx.doi.org/10.1002/tqem.20198

ROTHENBERG, S.; PIL, F. K.; MAXWELL, J. Lean, green, and the quest for superior environmental performance. Production and Operations Management, v. 10, n. 3, p. 228-243, 2001. http:// dx.doi.org/10.1111/j.1937-5956.2001.tb00372.x

SARKIS, J. Manufacturing's role in corporate environmental sustainability - Concerns for the new millennium, International Journal of Operations \& Production Management, v. 21, n. 5-6, p. 666-686, 2001. http:// dx.doi.org/10.1108/01443570110390390

SARKIS, J.; GONZALEZ-TORRE, P.; ADENSO-DIAZ, B. Stakeholder pressure and the adoption of environmental practices: the mediating effect of training. Journal of Operations Management, v. 28, p. 163-176, 2010. http://dx.doi.org/10.1016/j.jom.2009.10.001

SCHERRER-RATHJE, M.; BOYLE, T. A.; DEFLORIN, P. Lean, take two! Reflections from the second attempt at lean implementation. Business Horizons, v. 52, p. 79-88, 2009. http://dx.doi.org/10.1016/j. bushor.2008.08.004

SCHONBERGER, R. J. Japanese production management: an evolution - with mixed success. Journal of Operations Management, v. 25, p. 403-419, 2007. http://dx.doi. org/10.1016/j.jom.2006.04.003
SHAH, R.; WARD, P. T. Defining and developing measures of lean production. Journal of Operations Management, v. 25, p. 785-805, 2007. http://dx.doi.org/10.1016/j. jom.2007.01.019

SHAH, R.; WARD, P. T. Lean manufacturing: context, practice bundles, and performance. Journal of Operations Management, v. 21, p. 129-149, 2003. http://dx.doi.org/10.1016/S0272-6963(02)00108-0

SIMPSON, D. F.; POWER, D. J. Use the supply relationship to develop lean and green suppliers. Supply Chain Management: An International Journal, v. 10, n. 1, p. 60-68, 2005. http://dx.doi. org/10.1108/13598540510578388

SOSIK, J. J.; KAHAI, S. S.; PIOVOSO, M. J. Silver bullet or voodoo statistics?: A primer for using least squares data analytic technique in group and organization research. Group \& Organization Management, v. 35, n. 5, p. 5-36, 2009. http://dx.doi. org/10.1177/1059601108329198

SYNODINOS, N. E. The "art" of questionnaire construction: some important considerations for manufacturing studies. Integrated Manufacturing Systems, v. 14, n. 3, p. 221-237, 2003. http://dx.doi. org/10.1108/09576060310463172

VAIS, A. et al. "Lean and green" at a Romanian secondary tissue paper and board mill - putting theory into practice. Resources Conservation \& Recycling, v. 46, p. 44-74, 2006. http://dx.doi.org/10.1016/j. resconrec.2005.06.005

WILKINSON, A.; HILL, M.; GOLLAN, P. The sustainability debate. International Journal of Operations \& Production Management, v. 21, n. 12, p. 1492-1502, 2001. http://dx.doi.org/10.1108/01443570110410865

WOMACK, J. P.; JONES, D. T.; ROOS, D. The machine that changed the world. New York: Rawson Associates, 1990.

WOMACK, P. J.; JONES, D. T.; ROOS, D. A máquina que mudou o mundo. Rio de Janeiro: Elsevier, 2004.

WU, H.; DUNN, S.C. Environmentally responsible logistics systems. International Journal of Physical Distribution \& Logistics Management, v. 25, n. 2, p. 20-37, 2005. http://dx.doi.org/10.1108/09600039510083925

YANG, C. et al. Mediated effect of environmental management on manufacturing competitiveness: an empirical study. International Journal of Production Economics, v. 123, p. 210-220, 2010. http://dx.doi. org/10.1016/j.ijpe.2009.08.017

YANG, M. G.; HONG, P.; MODI, S. B. Impact of lean manufacturing and environmental management on business performance: an empirical study of manufacturing firms. International Journal of Production Economics, v. 129, n. 2, p. 251-261, 2011. http://dx.doi.org/10.1016/j. ijpe.2010.10.017 

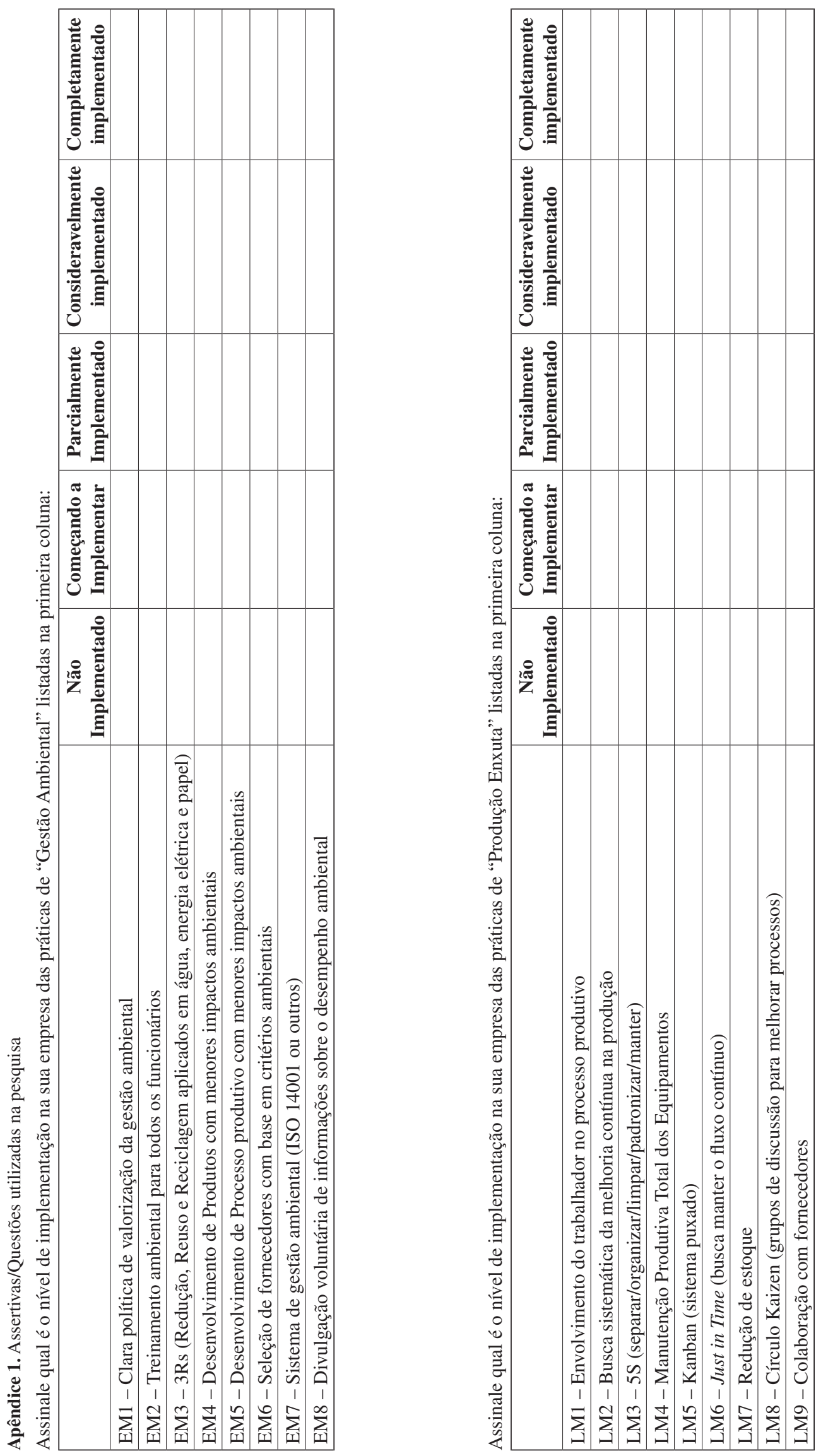